\title{
Very recent HIV infection accompanied by Pneumocystis jirovecii pneumonia and Mycobacterium avium complex immune reconstitution inflammatory syndrome: a case report
}

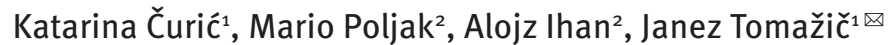

\begin{abstract}
A small proportion of HIV-infected patients rapidly progress to AIDS; indeed, some individuals have been known to progress to AIDS within a year after primary infection. Pneumonia caused by Pneumocystis jirovecii (PCP) is the most frequent AIDS-defining illness. However, PCP can also rarely occur during primary HIV infection as a result of the severe immunosuppression that may accompany the early stage of HIV infection. Immune reconstitution inflammatory syndrome (IRIS) comprises two distinct syndromes: paradoxical IRIS and unmasking IRIS. Infections with Mycobacterium avium complex during antiretroviral therapy are almost always localized and related to IRIS. We describe an unusual case of PCP and Mycobacterium avium complex-IRIS that occurred less than 3 months after primary HIV infection.
\end{abstract}

Keywords: HIV, immune reconstitution inflammatory syndrome, IRIS, Pneumocystits jirovecii, Mycobacterium avium complex

Received: 14 May 2016 | Returned for modification: 29 May 2016 | Accepted: 11 June 2016

\section{Case report}

A 34-year-old otherwise healthy woman presented to our institution in May 2013 with a 3-week history of dry cough and malaise, later accompanied by progressive shortness of breath, high-grade fever, and nausea. Her general practitioner performed chest radiographs, which showed diffuse bilateral infiltrates in the middle and lower lung fields, but empirical therapy for suspected atypical pneumonia with azithromycin was without benefit.

There was no history of intravenous drug use, prior sexually transmitted diseases, or blood transfusions. She tested negative for HIV in 2008, as did her ex-boyfriend. The patient reported several unprotected incidents of sexual intercourse with different male partners in the Philippines at the beginning January 2013 followed by a transient 2-week febrile flu-like illness. At presentation, the patient was febrile $\left(39.3^{\circ} \mathrm{C}\right)$ with oxygen saturation of $94 \%$ on room air, respiration rate 60 breaths/minute, tachycardia $116 \mathrm{bpm}$, and blood pressure 128/83 $\mathrm{mmHg}$, but otherwise unremarkable physical examination.

Blood tests revealed elevated C-reactive protein $(186 \mathrm{mg} / \mathrm{l})$, sedimentation rate $(100 \mathrm{~mm} / \mathrm{h})$, and lactic dehydrogenase $(7.85$ $\mu \mathrm{kat} / \mathrm{l})$ with a normal white blood cell count. $(1,3)$-beta-D glucan was positive.

High-resolution computer tomography showed ground glass opacities in the lingula and lower lung fields. HIV-1/2/O screening assays were highly reactive, and HIV-1 Western blot and HIV1/2 immunoblot tests confirmed HIV-1 infection. Direct immunofluorescent assay of induced sputum confirmed Pneumocystis jirovecii infection. Parenteral trimethoprim/sulfamethoxazole (TMP/SMX) with corticosteroids was initiated. HIV-1 viral load was extremely high $(1,232,000$ copies of HIV-1 RNA/mL) and CD4 T-cell count was 110 cells $/ \mathrm{mm}^{3}(6 \%)$. Plasma cytokine concentrations were high, interleukin-6 reached 427 pg/ml, and interleukin-8 61 pg/ml. HIV genotyping revealed HIV-1 subtype A1 without evidence of transmitted HIV drug resistance. HLA B5701 was negative, the HIV tropism assay showed CCR5 tropic viruses only, and the Aware
BED EIA HIV-1 Incidence Test (Calypte Biomedical Corporation, Portland, Oregon) indicated recent HIV infection. HLA typing using sequence-specific oligonucleotide-primed PCR and Luminex technology identified HLA-A*01, HLA-A ${ }^{\star} 03$, HLA-B ${ }^{\star} 27$, and HLA$B^{\star} 35$ : all not associated with rapid development of HIV-related immunodeficiency; in contrast, HLA-B ${ }^{\star} 27$ is associated with slow disease progression. Antiretroviral therapy (ART) with tenofovir/emtricitabine and raltegravir was initiated. After 21 days of treatment, the patient was discharged on prophylactic doses of TMP/SMX. Chest radiographs were nearly normal, the HIV-1 viral load drastically declined (427 copies/ml), the CD4 count rose to 250 cells $/ \mathrm{mm}^{3}$, and cytokine concentrations normalized. After 25 days, the patient was readmitted with progressive dyspnea, dry cough, chest pain, headache, subfebrile temperatures, and persistent rash. Clinically, she was tachypneic (40/min), tachycardic $(128 / \mathrm{min})$, and subfebrile $\left(37.3^{\circ} \mathrm{C}\right)$; a rash was hardly noticeable, and on auscultation expiratory wheezes on the right side and prolonged expirium were heard. Except for elevated C-reactive protein $(84 \mathrm{mg} / \mathrm{l})$, blood tests were unremarkable. The HIV-1 viral load further declined to 188 copies/ml and the CD4 T cell count was 356 cells $/ \mathrm{mm}^{3}$ (13\%). Chest radiographs showed a consolidation above the right hilum. Oxygen therapy and TMP/SMX with corticosteroids was restarted. Bronchoalveolar lavage was negative for P. jirovecii, Legionella pneumophila, Mycobacterium tuberculosis, and other pathogenic bacteria and fungi. At that time, diagnosis of lower respiratory system infection in the setting of PCP-related immune reconstitution inflammatory syndrome (PCP-IRIS) was most probable due to negative microbiological tests and rapid restoration of immunity. After 2 weeks of therapy, the intensity of consolidation regressed and the patient was discharged on prophylactic doses of TMP/SMX and ART. Twenty days after bronchoscopy, bronchoalveolar lavage was positive (40 colonies) for Mycobacterium avium complex (MAC). The patient successfully recovered after 14 months of MAC-IRIS treatment with clarithromycin and ethambutol with $\mathrm{CD} 4$ counts of 324 cells $/ \mathrm{mm}^{3}$ and a viral load of $<40$ copies $/ \mathrm{ml}$. 


\section{Discussion}

The suspicion that our patient's condition was a rapidly progressing HIV infection and not a primary HIV infection was supported by several facts: (i) the flu-like illness after traveling to the Philippines was probably primary HIV infection; (ii) the patient is infected with HIV-1 subtype A1, which is rarely found in Slovenia (1); and (iii) a high HIV-1 viral load and low CD4 T cells with rapid virological/immunological improvement after ART initiation. After primary HIV infection of mild intensity, profound immunosuppression occurred, leading to greater susceptibility to $P$. jirovecii infection-which is a characteristic pathogen in the late stages of AIDS and is only rarely seen in the early phase of HIV infection.

There is no consensus on optimal timing for initiation of ART in patients with acute opportunistic infection (2). In patients with PCP, growing evidence supports starting ART early rather than waiting for completion of PCP treatment. A randomized controlled trial compared early versus delayed ART in 282 HIV-infected patients diagnosed with opportunistic infection, of whom $63 \%$ had PCP (3). Early ART was associated with a significant decrease in AIDS progression and death without an increase in IRIS (3).

Initially we believed that a second episode of acute lower respiratory infection was related to the rare clinical entity PCP-IRIS due to rapid immunological restoration and a drop in HIV RNA after 6 weeks of ART. Subsequently, we unexpectedly isolated MAC from bronchoalveolar lavage but, because of the patient's improvement, MAC treatment was not initiated until repeated bronchoscopy showed a positive histology and culture. During the ART era, MAC infection has developed into a completely new disease. Previously, it had occurred as a disseminated disease in patients with massive immunodeficiency (4), but under ART it is mostly localized and related to IRIS that most often occurs within 3 months of initiating treatment (5), as in our patient; it is an unmasking of subclinical disease. MAC-IRIS can present with very diverse signs and symptoms: most often lymph nodes, the liver, or the gastrointestinal tract are involved, but essentially any organ could be involved, including the lungs (6).

HIV infection in our patient progressed very rapidly with two different opportunistic infections: PCP and, surprisingly, MACIRIS. Rapid progression of HIV infection into AIDS is linked to certain human as well as viral characteristics/markers. Interestingly, not a single HLA gene previously linked with rapid HIV progression (7) was identified in our patient. In contrast, a high HIV-1 viral load that does not fall dramatically after primary HIV infection, which is a strong marker of rapid HIV progression (8), was observed in our patient. The literature suggests that some rapid progressors are infected with rapidly replicating HIV strains and have viral population with higher heterogeneity than HIV viral controllers (8-10). Unfortunately, we were not in a position to measure these parameters in our patient. Although our patient experienced an unusual and challenging 1st year of her HIV-1 infection, she is currently doing well: after a favorable response to standard anti-MAC treatment, she is asymptomatic with normal chest radiographs, an unmeasurable HIV-1 viral load, and 318 CD4 T cells $/ \mathrm{mm}^{3}$.

\section{References}

1. Lunar MM, Židovec Lepej S, Abecasis AB, Tomažič J, Vidmar L, Karner P, et al. Short communication: prevalence of HIV type 1 transmitted drug resistance in Slovenia: 2005-2010. AIDS Res Hum Retroviruses. 2013;29:343-9.

2. Kaplan JE, Benson C, Holmes KK, Brooks JT, Pau A, Masur H, et al. Guidelines for prevention and treatment of opportunistic infections in HIV-infected adults and adolescents [internet]. Recommendations from CDC, the National Institutes of Health, and the HIV Medicine Association of the Infectious Diseases Society of America. [cited 2014 Mar 17]. Available from: http://www.cdc.gov/mmwr/preview/mmwrhtml/rr5804a1.htm.

3. Zolopa A, Andersen J, Powderly W, Sanchez A, Sanne I, Suckow C, et al. Early antiretroviral therapy reduces AIDS progression/death in individuals with acute opportunistic infections: a multicenter randomized strategy trial. PLoS ONE. 2009;4:e5575.

4. Jacobson MA, Hopewell PC, Yajko DM, Hadley WK, Lazarus E, Mohanty PK, et al. Natural history of disseminated Mycobacterium avium complex infection in AIDS. J Infect Dis. 1991;164:994-8.

5. Riddell J 4th, Kaul DR, Karakousis PC, Gallant JE, Mitty J, Kazanjian PH. Mycobacterium avium complex immune reconstitution inflammatory syndrome: long term outcomes. J Transl Med. 2007;5:50.

6. Gulati A, Singh S, Moussa R, Promnitz DA. Mycobacterium avium-intracellulare presenting as an endobronchial tumour due to immune reconstitution inflammatory syndrome. Int J STD AIDS. 2012;23:441-2.

7. Kaslow RA, Carrington M, Apple R, Park L, Muñoz A, Saah AJ. Influence of combinations of human major histocompatibility complex genes on the course of HIV-1 infection. Nat Med. 1996;2:405-11.

8. Khanlou H, Salmon-Ceron D, Sicard D. Characteristics of rapid progressors in HIV infection. Ann Med Interne (Paris). 1997;148:163-6.

9. Sandonís V, Casado C, Alvaro T, Pernas M, Olivares I, García S, et al. A combination of defective DNA and protective host factors are found in a set of HIV-1 ancestral LTNPs. Virology. 2009;391:73-82.

10. Wang B, Mikhail M, Dyer WB, Zaunders JJ, Kelleher AD, Saksena NK. First demonstration of a lack of viral sequence evolution in a nonprogressor, defining replication-incompetent HIV-1 infection. Virology. 2003;312:135-50. 